\title{
LIFTINGS OF THE ELEMENTARY GROUP OVER ASSOCIATIVE RINGS
}

\author{
BENJAMIN KLOPSCH \\ Mathematical Institute, University of Oxford, Oxford OX1 3LB, UK \\ e-mail:klopsch@maths.ox.ac.uk
}

(Received 17 July, 1998)

\begin{abstract}
Let $R$ be an associative ring with 1, and let $I$ be a nilpotent two-sided ideal of $R$. Assume further that there exists $z \in Z(R)$ such that $z, z^{2}-1 \in R^{*}$. Let $m \in \mathbb{N}$ with $m \geq 3$. In this paper we describe all liftings of the elementary group $\mathbf{E}_{m}(R / I)$ to the general linear group $\mathbf{G L}_{m}(R)$, i.e. all splittings of the natural projection $\mathbf{E}_{m}(R)+\mathbf{M}_{m}(I) \rightarrow \mathbf{E}_{m}(R / I)$.
\end{abstract}

1991 Mathematics Subject Classification. 20H25.

1. Introduction. Let $R$ be an associative ring with 1 , and let $I$ be a nilpotent two-sided ideal of $R$. We denote by $R^{*}$ the group of units of $R$ and by $Z(R)$ the centre of $R$. Let $m \in \mathbb{N}$ with $m \geq 3$. We denote by $\mathbf{M}_{m}(R)$ the set of $m \times m$ matrices over $R$, and we write $\mathbf{G L}_{m}(R)$ for the set of invertible $m \times m$ matrices over $R$. We denote by Id the $m \times m$ identity matrix over $R$. For $i, j \in \mathbb{N}$ we write $\delta_{i j}$ for the Kronecker delta. For all $i, j \in \mathbb{N}_{\leq m}$ let $E_{i j}:=\left(\delta_{i k} \delta_{j l}\right)_{k l} \in \mathbf{M}_{m}(R)$. We recall that the elementary group over $R$ is defined as

$$
\left.\mathbf{E}_{m}(R):=\left\langle\mathrm{Id}+a E_{i j}\right| a \in R \text { and } i, j \in \mathbb{N}_{\leq m} \text { with } i \neq j\right\rangle \leq \mathbf{G L}_{m}(R) .
$$

The purpose of this paper is to describe all splittings of the natural projection $\mathbf{E}_{m}(R)+\mathbf{M}_{m}(I) \rightarrow \mathbf{E}_{m}(R / I)$. We recall that a monomorphism $v: \mathbf{E}_{m}(R / I) \rightarrow$ $\mathbf{G L}_{m}(R)$ is said to be standard if it is induced by a ring monomorphism modulo an inner automorphism, i.e. if there exist a ring monomorphism $\xi: R / I \rightarrow R$ and a matrix $C \in \mathbf{G L}_{m}(R)$ such that for all $A=\left(A_{i j}\right) \in \mathbf{E}_{m}(R / I)$ we have $A v=C^{-1}\left(A_{i j} \xi\right) C$. We prove the following result.

THeOREM 1. (Liftings of the elementary group). Let $R$ be an associative ring with 1 , and let $I$ be a nilpotent two-sided ideal of $R$. Assume further that there exists $z \in Z(R)$ such that $z, z^{2}-1 \in R^{*}$. Let $\mu: \mathbf{E}_{m}(R)+\mathbf{M}_{m}(I) \rightarrow \mathbf{E}_{m}(R / I)$ denote the natural projection. Then every group monomorphism $v: \mathbf{E}_{m}(R / I) \rightarrow \mathbf{G L}_{m}(R)$ with $\nu \mu=\mathrm{id}_{\mathbf{E}_{m}(R / I)}$ is standard.

Under the given assumptions, Theorem 1 asserts in particular that there exists a splitting of $\mathbf{E}_{m}(R)+\mathbf{M}_{m}(I) \rightarrow \mathbf{E}_{m}(R / I)$ if and only if there exists a splitting of $R \rightarrow R / I$. We emphasise that the conclusion of the theorem does not follow without assuming the existence of $z \in Z(R)$ with $z, z^{2}-1 \in R^{*}$. As explained below, the group $\mathrm{SL}_{3}(\mathbb{Z} / 2 \mathbb{Z})$ lifts to $\mathrm{SL}_{3}(\mathbb{Z} / 4 \mathbb{Z})$, but clearly the ring $\mathbb{Z} / 2 \mathbb{Z}$ does not lift to $\mathbb{Z} / 4 \mathbb{Z}$.

It seems worth noting that results like Theorem 1 have interesting applications, for example in the theory of cohomology. In [4] Sah benefits from proving that for natural numbers $n \geq 2$ and prime numbers $p$ the special linear group $\mathbf{S L}_{n}(\mathbb{Z} / p \mathbb{Z})$ 
does not lift to $\mathbf{S L}_{n}\left(\mathbb{Z} / p^{2} \mathbb{Z}\right)$ unless $(n, p) \in\{(2,2),(3,2),(2,3)\}[\mathbf{4}$, Section II, Theorem 7]. Replacing $\mathbb{Z}$ by the ring of integers of an algebraic number field $K$ over $\mathbb{Q}$ and assuming that $p$ is unramified in $K$, he obtains in a second paper a generalisation of this result [5]. Note that both versions are essentially covered by Theorem 1, which excludes the cases $n=2$ and $p=2$. Moreover we do not require any assumptions concerning ramification. It would be interesting to find out whether our theorem gives rise to similar applications in the theory of cohomology.

We would also like to point out that the problem which we solve in Theorem 1 is related to questions which are usually investigated in the isomorphism theory for linear groups over rings. We do not wish to elaborate on this, but refer the reader to [1] for an introduction to the subject. Petechuk has published a number of papers in this area. In [2] and [3] he describes the homomorphisms between various linear and projective linear groups, where his results depend on certain conditions imposed upon the underlying rings (such as commutativity or 2 being invertible). Although our theorem deals with a very similar situation, there seems to be no direct link with his results.

The rest of the paper is organised as follows. In Section 2 we recall some basic facts about elementary matrices. In Section 3 we derive two key lemmata. Finally, in Section 4 we prove Theorem 1 .

Standing Assumptions and Further Notation. Throughout the rest of the paper let $R$ be an associative ring with 1 . We assume further that there exists $z \in Z(R)$ such that $z, z^{2}-1 \in R^{*}$. Let $r \in \mathbb{N}$, and let $I$ be a two-sided ideal of $R$ with $I^{r}=0$. We write $\bar{a}$ for the image of $a \in R$ under the natural projection $R \rightarrow R / I$. Let $m \in \mathbb{N}$ with $m \geq 3$, and let $\mathbb{N}_{\leq m}:=\{n \in \mathbb{N} \mid n \leq m\}$. Let $\mu: \mathbf{M}_{m}(R) \rightarrow \mathbf{M}_{m}(R / I)$ denote the natural projection. We denote by $\mathbf{D}_{m}(R)$ the set of diagonal $m \times m$-matrices over $R$. Furthermore, for all $b \in R^{*}$ and all $i, j \in \mathbb{N}_{\leq m}$ with $i \neq j$ we define $D_{i j}(b):=\mathrm{Id}+(b-1) E_{i i}+\left(b^{-1}-1\right) E_{j j} \in \mathbf{G L}_{m}(R)$, and write

$$
\left.\widehat{\mathbf{D}}_{m}(R):=\left\langle D_{i j}(b)\right| b \in R^{*} \text { and } i, j \in \mathbb{N}_{\leq m} \text { with } i \neq j\right\rangle \leq \mathbf{G L}_{m}(R) \cap \mathbf{D}_{m}(R) .
$$

We use a similar notation for (sets of) matrices over $R / I$ or $I$. If $G$ is a semigroup and if $x, y, z \in G$ such that $y$ and $z$ are invertible, we write $x^{y}:=y^{-1} x y$ and $[y, z]:=y^{-1} z^{-1} y z$.

\section{Some basic facts.}

Lemma 2.1. Let $a \in R$. Then $a \in R^{*}$ if and only if $\bar{a} \in(R / I)^{*}$.

Proof. This is a consequence of $I$ being nilpotent.

We note that in particular $1+I$ is a subgroup of $R^{*}$. Moreover, a similar argument shows that $\operatorname{Id}+\mathbf{M}_{m}(I)$ is a subgroup of $\mathbf{G L}_{m}(R)$.

Lemma 2.2. Let $i, j, k, l \in \mathbb{N}_{\leq m}$. Then the following hold.

(a) $E_{i j} E_{k l}=\delta_{j k} E_{i l}$.

(b) If $i \neq k$, then the map $R \rightarrow \mathbf{G L}_{m}(R), a \mapsto \mathrm{Id}+a E_{i k}$ is a group monomorphism from $(R,+)$ into $\mathbf{G L}_{m}(R)$. 
(c) If $i, j, k$ are pairwise distinct, and if $a, b \in R^{*}$ and $c, d \in R$, then

$$
\begin{aligned}
{\left[a\left(\operatorname{Id}+c E_{i j}\right), b\left(\operatorname{Id}+d E_{j k}\right)\right] } & =[a, b] \operatorname{Id}+\left([a, b] d-d^{a}[a, b]\right) E_{j k}+\left([a, b] c^{b}-c[a, b]\right) E_{i j} \\
& +\left([a, b] c^{b} d-c[a, b] d+c d^{a}[a, b]\right) E_{i k} .
\end{aligned}
$$

(d) If $i \neq j$, then for every $b \in R^{*}$ we have

$$
D_{i j}(b)=\left(\operatorname{Id}+(b-1) E_{i j}\right)\left(\mathrm{Id}+E_{j i}\right)\left(\mathrm{Id}+\left(b^{-1}-1\right) E_{i j}\right)\left(\mathrm{Id}-b E_{j i}\right) .
$$

Proof. These assertions are easily checked by direct computation.

COROllary 2.3. The group $\widehat{\mathbf{D}}_{m}(R)$ is contained in $\mathbf{E}_{m}(R)$.

Proof. This follows from part (d) of Lemma 2.2.

3. Two lemmata. In this section we begin to analyse the splittings of $\mathbf{E}_{m}(R)+\mathbf{M}_{m}(I) \rightarrow \mathbf{E}_{m}(R / I)$. In Lemma 3.1 we show that diagonal matrices lift essentially to diagonal matrices, and in Lemma 3.2 we prove that elementary matrices lift essentially to elementary matrices. We use these two results in Section 4 to establish Theorem 1.

Lemma 3.1. (Liftings of diagonal matrices). Let $v: \widehat{\mathbf{D}}_{m}(R / I) \rightarrow \mathbf{G L}_{m}(R)$ be a group monomorphism with $\nu \mu=\mathrm{id}_{\widehat{\mathbf{D}}_{m}(R / I)}$. Then there exists $C \in \mathrm{Id}+\mathbf{M}_{m}(I)$ such that $\widetilde{v}: \widehat{\mathbf{D}}_{m}(R / I) \rightarrow \mathbf{G L}_{m}(R), D \mapsto C^{-1}(D v) C$ has the following properties.

(a) The map $\widetilde{v}$ is a group monomorphism with $\widetilde{v} \mu=\mathrm{id}_{\widehat{\mathbf{D}}_{m}(R / I)}$.

(b) For all $b \in R^{*}$ and all $i, j \in \mathbb{N}_{\leq m}$ with $i \neq j$ we have $D_{i j}(\bar{b}) \widetilde{v} \in \mathbf{D}_{m}(R)$.

Proof. We argue by induction on $r$. The case $r=1$ is clear, since $v=\mathrm{id}$.

Induction Step: $r>1$. We prove the result in four steps.

(i) We have $z, z^{2}-1, z-1, z^{-1}-1, z-z^{-1} \in R^{*}$.

Proof. This is clear, since by standing assumption we have $z, z^{2}-1 \in R^{*}$.

(ii) Without loss of generality we may assume that for all $b \in R^{*}$ and all $i, j \in \mathbb{N}_{\leq m}$ with $i \neq j$,

$$
D_{i j}(\bar{b}) v \in \mathbf{D}_{m}(R)+\mathbf{M}_{m}\left(I^{r-1}\right) .
$$

Proof. Let $\lambda: \mathbf{M}_{m}(R) \rightarrow \mathbf{M}_{m}\left(R / I^{r-1}\right)$ and $\widehat{\mu}: \mathbf{M}_{m}\left(R / I^{r-1}\right) \rightarrow \mathbf{M}_{m}(R / I)$ denote the natural projections. Put $\widehat{v}:=v \lambda: \widehat{\mathbf{D}}_{m}(R / I) \rightarrow \mathbf{G L}_{m}\left(R / I^{r-1}\right)$. Replacing $(R, I, v, \mu, \ldots)$ by $\left(R / I^{r-1}, I / I^{r-1}, \widehat{v}, \widehat{\mu}, \ldots\right)$, we now apply the induction hypothesis to obtain a matrix $\widehat{C} \in \mathrm{Id}+\mathbf{M}_{m}\left(I / I^{r-1}\right)$ such that $\widehat{C}^{-1}\left(D_{i j}(\bar{b}) \widehat{v}\right) \widehat{C} \in \mathbf{D}_{m}\left(R / I^{r-1}\right)$ for all $b \in R^{*}$ and all $i, j \in \mathbb{N}_{\leq m}$ with $i \neq j$. By the remark following Lemma 2.1, we can lift $\widehat{C}$ to some $C \in \operatorname{Id}+\mathbf{M}_{m}(I)$ so that $C^{-1}\left(D_{i j}(\bar{b}) v\right) C \in \mathbf{D}_{m}(R)+\mathbf{M}_{m}\left(I^{r-1}\right)$ for all $b \in R^{*}$ and all $i, j \in \mathbb{N}_{\leq m}$ with $i \neq j$. 
(iii) Let $b \in R^{*}$, and let $i, j \in \mathbb{N}_{\leq m}$ with $i \neq j$. Then for all $k, l \in \mathbb{N}_{\leq m} \backslash\{i, j\}$ with $k \neq l$ the $(k, l)$-entry of $D_{i j}(\bar{b})$ v equals zero.

Proof. Let $k, l \in \mathbb{N}_{\leq m} \backslash\{i, j\}$ with $k \neq l$. By (ii), we find $D_{B}, D_{Z} \in \mathbf{D}_{m}(I)$ such that

$$
\begin{aligned}
& B:=D_{i j}(\bar{b}) v-D_{i j}(b)-D_{B} \in \mathbf{M}_{m}\left(I^{r-1}\right), \\
& Z:=D_{k l}(\bar{z}) v-D_{k l}(z)-D_{Z} \in \mathbf{M}_{m}\left(I^{r-1}\right) .
\end{aligned}
$$

Since $D_{i j}(\bar{b})$ and $D_{k l}(\bar{z})$ commute, so do $D_{i j}(\bar{b}) v$ and $D_{k l}(\bar{z}) v$, hence so do $D_{i j}(b)+D_{B}+B$ and $D_{k l}(z)+D_{Z}+Z$. We have

$$
\left(D_{i j}(b)+D_{B}+B\right)\left(D_{k l}(z)+D_{Z}+Z\right)=\left(D_{k l}(z)+D_{Z}+Z\right)\left(D_{i j}(b)+D_{B}+B\right) .
$$

Recalling that $I^{r}=0$ and $k \neq l$, this implies that the $(k, l)$-entries of $D_{i j}(b) Z+B D_{k l}(z)$ and $Z D_{i j}(b)+D_{k l}(z) B$ are equal. This gives

$$
Z_{k l}+B_{k l} z^{-1}=Z_{k l}+z B_{k l}
$$

Recalling that $z \in Z(R)$ and $z-z^{-1} \in R^{*}$, we obtain $B_{k l}=0$.

(iv) There exists $C \in \mathrm{Id}+\mathbf{M}_{m}(I)$ such that the map $\widetilde{v}: \widehat{\mathbf{D}}_{m}(R / I) \rightarrow \mathbf{G L}_{m}(R)$, $D \mapsto C^{-1}(D v) C$ has properties (a) and (b) as stated in the lemma.

Proof. We claim that for all $n \in\{0,1, \ldots, m-1\}$ we find $C_{n} \in \mathrm{Id}+\mathbf{M}_{m}(I)$ such that for all $b \in R^{*}$ and all $i, j, k, l \in \mathbb{N}_{\leq m}$ with $i \neq j, k \neq l$ and $\min \{k, l\} \leq n$ the $(k, l)$ entry of $C_{n}^{-1}\left(D_{i j}(\bar{b}) v\right) C_{n}$ is zero. Then $C:=C_{m-1}$ has the desired properties.

So let $n \in\{0,1, \ldots, m-1\}$. We argue by induction. In the case $n=0$ there is nothing to show.

Induction Step: $n \geq 1$. By induction hypothesis, we may assume without loss of generality that for all $b \in R^{*}$ and all $i, j, k, l \in \mathbb{N}_{\leq m}$ with $i \neq j, k \neq l$ and $\min \{k, l\} \leq n-1$ the $(k, l)$-entry of $D_{i j}(\bar{b}) v$ is zero. By (ii), we find $D_{Z} \in \mathbf{D}_{m}(I)$ such that

$$
Z:=D_{n, n+1}(\bar{z}) v-D_{n, n+1}(z)-D_{Z} \in \mathbf{M}_{m}\left(I^{r-1}\right) .
$$

Moreover, from (iii) we know that non-zero non-diagonal entries of $D_{n, n+1}(\bar{z}) v$ may only occur in the $n$-th or $(n+1)$-th row or column. We define

$$
\begin{aligned}
& C_{n}^{(1)}:=\mathrm{Id}-\sum\left\{(z-1)^{-1} Z_{n k} E_{n k} \mid n+2 \leq k \leq m\right\}, \\
& C_{n}^{(2)}:=\mathrm{Id}+\sum\left\{Z_{k n}(z-1)^{-1} E_{k n} \mid n+2 \leq k \leq m\right\}, \\
& C_{n}^{(3)}:=\mathrm{Id}-\left(z-z^{-1}\right)^{-1} Z_{n, n+1} E_{n, n+1}, \\
& C_{n}^{(4)}:=\mathrm{Id}+\left(z-z^{-1}\right)^{-1} Z_{n+1, n} E_{n+1, n} .
\end{aligned}
$$

We put $C_{n}:=C_{n}^{(1)} C_{n}^{(2)} C_{n}^{(3)} C_{n}^{(4)} \in \mathrm{Id}+\mathbf{M}_{m}\left(I^{r-1}\right)$. Recalling Lemma 2.2, it is easy to check that conjugation by $C_{n}$ only changes matrix entries in positions $(k, n)$ and $(n, k)$, where $n+1 \leq k \leq m$, and moreover that all these entries are zero for $C_{n}{ }^{-1}\left(D_{n, n+1}(\bar{z}) \nu\right) C_{n}$. So, conjugating by $C_{n}$, we may assume without loss of generality 
that for all $k \in \mathbb{N}_{\leq m}$ with $k \geq n+1$ the $(k, n)$-entry and the $(n, k)$-entry of $D_{n, n+1}(\bar{z}) v$ are both zero.

Now let $b \in R^{*}$, and let $i, j, k, l \in \mathbb{N}_{\leq m}$ with $i \neq j, k \neq l$ and $\min \{k, l\} \leq n$. To finish the proof, we have to show that the $(k, l)$-entry of $D_{i j}(\bar{b}) v$ is zero. We know already that this is true if $\min \{k, l\} \leq n-1$. So let us assume that $\min \{k, l\}=n$. By (ii), we find $D_{B} \in \mathbf{D}_{m}(I)$ such that $B:=D_{i j}(\bar{b}) v-D_{i j}(b)-D_{B} \in \mathbf{M}_{m}\left(I^{r-1}\right)$. Since $D_{n, n+1}(\bar{z})$ and $D_{i j}(\bar{b})$ commute, so do $D_{n, n+1}(\bar{z}) v$ and $D_{i j}(b)+D_{B}+B=D_{i j}(\bar{b}) v$. Recalling that $I^{r}=0$ and $k \neq l$, we deduce that the $(k, l)$-entries of $\left(D_{n, n+1}(\bar{z}) v\right) B$ and $B\left(D_{n, n+1}(\bar{z}) v\right)$ are equal. We distinguish four cases.

Case 1: $(k, l)=(n, n+1)$. Then $z B_{n, n+1}=B_{n, n+1} z^{-1}$. Recalling that $z \in Z(R)$ and $z-z^{-1} \in R^{*}$, we obtain $B_{k l}=B_{n, n+1}=0$.

Case 2: $k=n$ and $l \geq n+2$. Then $z B_{n l}=B_{n l}$. Recalling that $z-1 \in R^{*}$, we obtain $B_{k l}=B_{n l}=0$.

Case 3: $(k, l)=(n+1, n)$. This is similar to Case 1 .

Case 4: $k \geq n+2$ and $l=n$. This is similar to Case 2 .

This finishes the proof of the lemma.

Lemma 3.2. (Liftings of elementary matrices). Let $v: \mathbf{E}_{m}(R / I) \rightarrow \mathbf{G L}_{m}(R)$ be a group monomorphism with $\nu \mu=\operatorname{id}_{\mathbf{E}_{m}(R / I)}$ and such that for all $b \in R^{*}$ and all $i, j \in \mathbb{N}_{\leq m}$ with $i \neq j$ we have $D_{i j}(\bar{b}) v \in \mathbf{D}_{m}(R)$.

Then for all $a \in R$ and all $i, j \in \mathbb{N}_{\leq m}$ with $i \neq j$ there exists $d_{i j}(a) \in I$ such that

$$
\left(\mathrm{Id}+\bar{a} E_{i j}\right) v=\mathrm{Id}+\left(a+d_{i j}(a)\right) E_{i j} .
$$

Proof. We argue by induction on $r$. The case $r=1$ is clear, since $v=\mathrm{id}$.

Induction Step: $r>1$. We divide the proof into seven steps.

(i) For all $d_{1} \in I$ and all $d_{2} \in I^{r-1}$ we have $\left(1+d_{1}\right)\left(1+d_{2}\right)=\left(1+d_{2}\right)\left(1+d_{1}\right)$.

Proof. This follows from $I^{r}=0$.

(ii) Without loss of generality we may assume that for all $a \in R$ and all $i, j \in \mathbb{N}_{\leq m}$ with $i \neq j$ there exists $d_{i j}(a) \in I$ such that

$$
\left(\mathrm{Id}+\bar{a} E_{i j}\right) v-\left(\mathrm{Id}+\left(a+d_{i j}(a)\right) E_{i j}\right) \in \mathbf{M}_{m}\left(I^{r-1}\right) .
$$

Proof. This follows by the induction hypothesis, just as claim (ii) did in the proof of Lemma 3.1.

(iii) Let $b \in R^{*}$, and let $i, j \in \mathbb{N}_{\leq m}$ with $i \neq j$. Let $n \in \mathbb{N}_{\leq m}$. Then the $(n, n)$-entries of $\left(\mathrm{Id}+E_{i j}\right) v$ and $\left(\mathrm{Id}+\bar{b}^{2} E_{i j}\right) v$ are equal.

Proof. From (DL), (i) and (ii) we have: for all $a \in R^{*}$ and all $k, l \in \mathbb{N}_{\leq m} \backslash\{n\}$ with $k \neq l$ the $(n, n)$-entries of $\left(\operatorname{Id}+E_{i j}\right) v$ and $\left(D_{k l}(\bar{a})\left(\operatorname{Id}+E_{i j}\right) D_{k l}\left(\bar{a}^{-1}\right)\right) v=$ $D_{k l}(\bar{a}) v\left(\mathrm{Id}+E_{i j}\right) v D_{k l}\left(\bar{a}^{-1}\right) v$ are equal. We distinguish three cases. 
Case 1: $n \notin\{i, j\}$. The result follows from

$$
\left(\mathrm{Id}+\bar{b}^{2} E_{i j}\right) v=D_{i j}(\bar{b}) v\left(\mathrm{Id}+E_{i j}\right) v D_{i j}\left(\bar{b}^{-1}\right) v .
$$

Case 2: $n=i$. Choose $k \in \mathbb{N}_{\leq m} \backslash\{i, j\}$. The result follows from

$$
\left(\mathrm{Id}+\bar{b}^{2} E_{i j}\right) v=D_{k j}\left(\bar{b}^{2}\right) v\left(\mathrm{Id}+E_{i j}\right) v D_{k j}\left(\bar{b}^{-2}\right) v .
$$

Case 3: $n=j$. This is similar to Case 2 .

(iv) Let $s, t, u, v \in \mathbb{N}_{\leq m}$ with $s \neq t, \quad t \neq u, \quad u \neq v, \quad v \neq s$. Let $a, b \in R$ and $X:=\mathrm{Id}+a E_{s t}, Y:=\mathrm{Id}+\bar{b} E_{u v} . B y$ (ii), we find $d_{A}, d_{B} \in I$ such that

$$
\begin{aligned}
A & :=X \mu \nu-X \in d_{A} E_{s t}+\mathbf{M}_{m}\left(I^{r-1}\right), \\
B & :=Y \mu \nu-Y \in d_{B} E_{u v}+\mathbf{M}_{m}\left(I^{r-1}\right) .
\end{aligned}
$$

By Lemma 2.2, the matrices $X$ and $Y$ commute, hence so do $X \mu \nu$ and $Y \mu \nu$, hence so do $X+A$ and $Y+B$. We also note that $A B=B A=0$. Thus we obtain

$$
\begin{aligned}
a E_{s t} B+A b E_{u v} & =(X+A)(Y+B)-X Y-A-B \\
& =(Y+B)(X+A)-Y X-A-B \\
& =B a E_{s t}+b E_{u v} A .
\end{aligned}
$$

(v) Let $a \in R$, and let $i, j \in \mathbb{N}_{\leq m}$ with $i \neq j$. We set $A:=\left(\operatorname{Id}+\bar{a} E_{i j}\right) \nu-$ $\left(\operatorname{Id}+a E_{i j}\right) \in \mathbf{M}_{m}(I)$. Then for all $k, l \in \mathbb{N}_{\leq m}$ with $k \neq l$ and $(k, l) \neq(i, j)$ we have $A_{k l}=0$.

Proof. Let $k, l \in \mathbb{N}_{\leq m}$ with $k \neq l$ and $(k, l) \neq(i, j)$. We consider four cases.

Case 1: $\{k, l\} \cap\{i, j\}=\emptyset$. Set $B:=\left(\operatorname{Id}+E_{l k}\right) v-\left(\operatorname{Id}+E_{l k}\right) \in \mathbf{M}_{m}(I)$. Then (iv) applied to $(s, t, u, v, b)=(i, j, l, k, 1)$ reads at the $(l, l)$-position: $0+0=0+A_{k l}$. Hence $A_{k l}=0$.

Case 2: $(k, l)=(j, i)$. Choose $n \in \mathbb{N}_{\leq m} \backslash\{i, j\}$. Set $B:=\left(\mathrm{Id}+E_{i n}\right) v-\left(\mathrm{Id}+E_{\text {in }}\right)$ $\in \mathbf{M}_{m}(I)$. Then (iv) applied to $(s, t, u, v, \bar{b})=(i, j, i, n, 1)$ reads at the $(j, n)$-position: $0+A_{j i}=0+0$. Hence $A_{k l}=A_{j i}=0$.

Case 3: $k=i$. Then $l \neq j$. Set $B:=\left(\operatorname{Id}+E_{l j}\right) v-\left(\operatorname{Id}+E_{l j}\right) \in \mathbf{M}_{m}(I)$, and set $Z:=\left(\operatorname{Id}+\bar{z}^{2} E_{l j}\right) v-\left(\operatorname{Id}+z^{2} E_{l j}\right) \in \mathbf{M}_{m}(I)$. Then (iv) applied to $(s, t, u, v, b)=$ $(i, j, l, j, 1)$ and $(s, t, u, v, b)=\left(i, j, l, j, z^{2}\right)$ gives at the $(i, j)$-position: $a B_{j j}+A_{i l}=$ $B_{i i} a+0$ and $a Z_{j j}+A_{i l} z^{2}=Z_{i i} a+0$. By (iii), $B_{j j}=Z_{j j}$. Hence $A_{i l}\left(z^{2}-1\right)=0$. Recalling that $z^{2}-1 \in R^{*}$, we obtain $A_{k l}=A_{i l}=0$.

Case 4: $k=j$ and $l \neq i$. This is similar to Case 3 .

(vi) Let $b \in R$, and let $i, j \in \mathbb{N}_{\leq m}$ with $i \neq j$. We set $B:=\left(\operatorname{Id}+\bar{b} E_{i j}\right) \nu-$ $\left(\mathrm{Id}+b E_{i j}\right) \in \mathbf{M}_{m}(I)$. Then $B_{k k}=B_{l l}$ for all $k, l \in \mathbb{N}_{\leq m}$.

Proof. Let $k, l \in \mathbb{N}_{\leq m}$. If $k=l$, there is nothing to prove. So assume $k \neq l$. We consider four cases.

Case 1: $\{k, l\} \cap\{i, j\}=\emptyset$. Put $A:=\left(\operatorname{Id}+E_{k l}\right) v-\left(\operatorname{Id}+E_{k l}\right) \in \mathbf{M}_{m}(I)$. Then (iv) for $(s, t, u, v, a)=(k, l, i, j, 1)$ reads at the $(k, l)$-position: $B_{l l}+0=B_{k k}+0$. Hence $B_{k k}=B_{l l}$. 
Case 2: $\{k, l\} \cap\{i, j\}=\{i\}$. Without loss of generality we may assume that $k=i$ and $l \neq j$. Put $A:=\left(\mathrm{Id}+E_{i l}\right) v-\left(\mathrm{Id}+E_{i l}\right) \in \mathbf{M}_{m}(I)$. Then (iv) for $(s, t, u, v, a)=$ $(i, l, j, l, 1)$ reads at the $(i, l)$-position: $B_{l l}+A_{i j} b=B_{i i}+0$. By $(\mathrm{v})$, we have $A_{i j}=0$. Hence $B_{k k}=B_{i i}=B_{l l}$.

Case 3: $\{k, l\} \cap\{i, j\}=\{j\}$. This is similar to Case 2 .

Case $4:\{k, l\}=\{i, j\}$. Since $m \geq 3$, this follows from Cases 2 and 3 .

(vii) Let $a \in R$, and let $i, j \in \mathbb{N}_{\leq m}$ with $i \neq j$. Then there exists $d_{i j}(a) \in I$ such that $\left(\mathrm{Id}+\bar{a} E_{i j}\right) v=\mathrm{Id}+\left(a+d_{i j}(a)\right) E_{i j}$.

Proof. Choose $k \in \mathbb{N}_{\leq m} \backslash\{i, j\}$. By (ii), (v) and (vi), we find $d_{1}, d_{2}, d_{3} \in I^{r-1}$ and $d_{4}, d_{5}, d_{6} \in I$ such that

$$
\begin{aligned}
& \left(\mathrm{Id}+E_{i k}\right) v=\left(1+d_{1}\right)\left(\mathrm{Id}+\left(1+d_{4}\right) E_{i k}\right), \\
& \left(\mathrm{Id}+\bar{a} E_{k j}\right) v=\left(1+d_{2}\right)\left(\mathrm{Id}+\left(a+d_{5}\right) E_{k j}\right), \\
& \left(\mathrm{Id}+\bar{a} E_{i j}\right) v=\left(1+d_{3}\right)\left(\mathrm{Id}+\left(a+d_{6}\right) E_{i j}\right) .
\end{aligned}
$$

By (i) and Lemma 2.2 (c), we also have

$$
\begin{aligned}
\left(\mathrm{Id}+\bar{a} E_{i j}\right) v= & {\left[\left(\mathrm{Id}+E_{i k}\right) v,\left(\operatorname{Id}+\bar{a} E_{k j}\right) v\right] } \\
= & {\left[\left(1+d_{1}\right)\left(\mathrm{Id}+\left(1+d_{4}\right) E_{i k}\right),\left(1+d_{2}\right)\left(\mathrm{Id}+\left(a+d_{5}\right) E_{k j}\right)\right] } \\
= & \mathrm{Id}+\left(a+d_{5}-\left(1+d_{1}\right)^{-1}\left(a+d_{5}\right)\left(1+d_{1}\right)\right) E_{k j} \\
& +\left(1+d_{4}\right)\left(1+d_{1}\right)^{-1}\left(a+d_{5}\right)\left(1+d_{1}\right) E_{i j} .
\end{aligned}
$$

Comparing components yields $a+d_{5}-\left(1+d_{1}\right)^{-1}\left(a+d_{5}\right)\left(1+d_{1}\right)=0$, and hence $\left(\mathrm{Id}+\bar{a} E_{i j}\right) v=\mathrm{Id}+\left(1+d_{4}\right)\left(a+d_{5}\right) E_{i j}$.

This finishes the proof of the lemma.

4. Proof of the theorem. In this section we show that every splitting of $\mathbf{E}_{m}(R)+\mathbf{M}_{m}(I) \rightarrow \mathbf{E}_{m}(R / I)$ is standard.

Proof of Theorem 1. Let $v: \mathbf{E}_{m}(R / I) \rightarrow \mathbf{G L}_{m}(R)$ be a group monomorphism with $\nu \mu=\mathrm{id}_{\mathbf{E}_{m}(R / I)}$. We divide the proof into four steps.

(i) By Lemma 3.1, we may assume without loss of generality that for all $b \in R^{*}$ and all $i, j \in \mathbb{N}_{\leq m}$ with $i \neq j$ we have $D_{i j}(\bar{b}) v \in \mathbf{D}_{m}(R)$. have

(ii) Without loss of generality we may assume that for all $i, j \in \mathbb{N}_{\leq m}$ with $i \neq j$ we

$$
\left(\mathrm{Id}+E_{i j}\right) v=\mathrm{Id}+E_{i j}
$$

Proof. For all $i \in \mathbb{N}_{\leq m}$ set $e_{i i}:=1$. By Lemma 3.2, we find, for all $i, j \in \mathbb{N}_{\leq m}$ with $i \neq j$, a (unique) $e_{i j} \in 1+I \leq R^{*}$ such that $\left(\mathrm{Id}+E_{i j}\right) v=\mathrm{Id}+e_{i j} E_{i j}$. Let $D \in \mathrm{Id}+\mathbf{D}_{m}(I)$ with diagonal entries $\left(e_{11}, e_{21}, e_{31}, \ldots, e_{m 1}\right)$. Then for all $i, j \in \mathbb{N}_{\leq m}$ with $i \neq j$ we have $D^{-1}\left(\mathrm{Id}+e_{i j} E_{i j}\right) D=\mathrm{Id}+e_{i 1}^{-1} e_{i j} e_{j 1} E_{i j}$. Hence it suffices to show that $e_{i 1}^{-1} e_{i j} e_{j 1}=1$ for all $i, j \in \mathbb{N}_{\leq m}$ with $i \neq j$. Indeed it is enough to prove

(a) for all pairwise distinct $i, j, k \in \mathbb{N}_{\leq m}$ we have $e_{i j} e_{j k}=e_{i k}$,

(b) for all $i, j \in \mathbb{N}_{\leq m}$ with $i \neq j$ we have $e_{i j} e_{j i}=1$. 
Recalling Lemma 2.2, we obtain for pairwise distinct $i, j, k \in \mathbb{N}_{\leq m}$,

$$
\begin{aligned}
\mathrm{Id}+e_{i j} e_{j k} E_{i k} & =\left[\mathrm{Id}+e_{i j} E_{i j}, \mathrm{Id}+e_{j k} E_{j k}\right] \\
& =\left[\left(\mathrm{Id}+E_{i j}\right) v,\left(\mathrm{Id}+E_{j k}\right) v\right] \\
& =\left[\mathrm{Id}+E_{i j}, \mathrm{Id}+E_{j k}\right] v \\
& =\left(\mathrm{Id}+E_{i k}\right) v=\mathrm{Id}+e_{i k} E_{i k},
\end{aligned}
$$

hence $e_{i j} e_{j k}=e_{i k}$. This proves claim (a).

Now let $i, j \in \mathbb{N}_{\leq m}$ with $i \neq j$. We choose $k \in \mathbb{N}_{\leq m} \backslash\{i, j\}$. Then using Lemma 2.2, we have

$$
\begin{aligned}
\mathrm{Id}+e_{j i} E_{j i} & =\left(\mathrm{Id}+E_{j i}\right) v \\
& =\left[\left[\mathrm{Id}+E_{j i}, \mathrm{Id}+E_{i k}\right],\left[\mathrm{Id}+E_{k j}, \mathrm{Id}+E_{j i}\right]\right] v \\
& =\left[\left[\left(\mathrm{Id}+E_{j i}\right) v,\left(\mathrm{Id}+E_{i k}\right) v\right],\left[\left(\mathrm{Id}+E_{k j}\right) v,\left(\mathrm{Id}+E_{j i}\right) v\right]\right] \\
& =\left[\left[\left(\mathrm{Id}+e_{j i} E_{j i}\right),\left(\mathrm{Id}+e_{i k} E_{i k}\right)\right],\left[\left(\mathrm{Id}+e_{k j} E_{k j}\right),\left(\mathrm{Id}+e_{j i} E_{j i}\right)\right]\right] \\
& =\mathrm{Id}+e_{j i}\left(e_{i k} e_{k j}\right) e_{j i} E_{j i} \\
& =\mathrm{Id}+e_{j i}\left(e_{i j} e_{j i}\right) E_{j i} .
\end{aligned}
$$

Since $e_{j i} \in R^{*}$, we have $e_{i j} e_{j i}=1$. This proves claim (b).

(iii) For all $i, j \in \mathbb{N}_{\leq m}$ with $i \neq j$ we find a (unique) map $\xi_{i j}: R / I \rightarrow R$ such that, for all $a \in R$,

$$
\left(\mathrm{Id}+\bar{a} E_{i j}\right) v=\mathrm{Id}+\left(\bar{a} \xi_{i j}\right) E_{i j}
$$

Put $\xi:=\xi_{12}$. Then for all $i, j \in \mathbb{N}$ with $i \neq j$ we have $\xi_{i j}=\xi$.

Proof. Since $m \geq 3$, it is enough to show that for all pairwise distinct $i, j, k \in \mathbb{N}_{\leq m}$ we have: $\xi_{i j}=\xi_{i k}=\xi_{j k}$. So let $a \in R$, and let $i, j, k \in \mathbb{N}_{\leq m}$ be pairwise distinct. Then we have

$$
\begin{aligned}
\mathrm{Id}+\left(\bar{a} \xi_{i j}\right) E_{i k} & =\left[\mathrm{Id}+\left(\bar{a} \xi_{i j}\right) E_{i j}, \mathrm{Id}+E_{j k}\right] \\
& =\left[\left(\mathrm{Id}+\bar{a} E_{i j}\right) v,\left(\mathrm{Id}+E_{j k}\right) v\right] \\
& =\left[\mathrm{Id}+\bar{a} E_{i j}, \mathrm{Id}+E_{j k}\right] v \\
& =\left(\mathrm{Id}+\bar{a} E_{i k}\right) v \\
& =\mathrm{Id}+\left(\bar{a} \xi_{i k}\right) E_{i k}
\end{aligned}
$$

and

$$
\begin{aligned}
\mathrm{Id}+\left(\bar{a} \xi_{i k}\right) E_{i k} & =\left(\mathrm{Id}+\bar{a} E_{i k}\right) v \\
& =\left[\mathrm{Id}+E_{i j}, \mathrm{Id}+\bar{a} E_{j k}\right] v \\
& =\left[\left(\mathrm{Id}+E_{i j}\right) v,\left(\mathrm{Id}+\bar{a} E_{j k}\right) v\right] \\
& =\left[\mathrm{Id}+E_{i j}, \mathrm{Id}+\left(\bar{a} \xi_{j k}\right) E_{j k}\right] \\
& =\mathrm{Id}+\left(\bar{a} \xi_{j k}\right) E_{i k} .
\end{aligned}
$$

This gives $\bar{a} \xi_{i j}=\bar{a} \xi_{i k}=\bar{a} \xi_{j k}$ as desired. 
(iv) The map $\xi$ is a ring monomorphism from $R / I$ into $R$. So $v$ is induced by $\xi$ and hence standard.

Proof. We need to show

(a) for all $a_{1}, a_{2} \in R$ we have $\left(\overline{a_{1}}+\overline{a_{2}}\right) \xi=\overline{a_{1}} \xi+\overline{a_{2}} \xi$,

(b) for all $a_{1}, a_{2} \in R$ we have $\left(\overline{a_{1} a_{2}}\right) \xi=\overline{a_{1}} \xi \overline{a_{2}} \xi$.

Let $a_{1}, a_{2} \in R$. Then we have

$$
\begin{aligned}
\mathrm{Id}+\left(\overline{a_{1}}+\overline{a_{2}}\right) \xi E_{12} & =\left(\operatorname{Id}+\left(\overline{a_{1}}+\overline{a_{2}}\right) E_{12}\right) v \\
& =\left(\operatorname{Id}+\overline{a_{1}} E_{12}\right) v\left(\mathrm{Id}+\overline{a_{2}} E_{12}\right) v \\
& =\left(\operatorname{Id}+\left(\overline{a_{1}} \xi\right) E_{12}\right)\left(\mathrm{Id}+\left(\overline{a_{2}} \xi\right) E_{12}\right) \\
& =\operatorname{Id}+\left(\overline{a_{1}} \xi+\overline{a_{2}} \xi\right) E_{12} .
\end{aligned}
$$

This gives (a). Furthermore we have

$$
\begin{aligned}
\mathrm{Id}+\left(\overline{a_{1} a_{2}}\right) \xi E_{12} & =\left(\mathrm{Id}+\overline{a_{1} a_{2}} E_{12}\right) v \\
& =\left[\left[\mathrm{Id}+\overline{a_{1}} E_{12}, \mathrm{Id}+E_{23}\right],\left[\mathrm{Id}+E_{31}, \mathrm{Id}+\overline{a_{2}} E_{12}\right]\right] v \\
& =\left[\left[\mathrm{Id}+\left(\overline{a_{1}} \xi\right) E_{12}, \mathrm{Id}+E_{23}\right],\left[\mathrm{Id}+E_{31}, \mathrm{Id}+\left(\overline{a_{2}} \xi\right) E_{12}\right]\right] \\
& =\mathrm{Id}+\left(\overline{a_{1}} \xi\right)\left(\overline{a_{2}} \xi\right) E_{12} .
\end{aligned}
$$

This gives (b) and finishes the proof of the theorem.

AcKnowledgements. This work is partly based on the author's M.Sc. thesis, University of East Anglia, 1996. He thanks his supervisor Professor Alexander Zalesskii and the referee for their helpful suggestions.

\section{REFERENCES}

1. A. J. Hahn and O. T. O'Meara, The classical groups and K-theory (Springer-Verlag, 1989)

2. V. I. Petechuk, Homomorphisms of linear groups over rings, Mat. Zametki 45 (1989), no. 2, 83-94, 142; translation: Math. Notes 45 (1989), no. 1-2, 144-151.

3. V. I. Petechuk, Homomorphisms of linear groups over commutative rings, Mat. Zametki 46 (1989), no. 5, 50-61, 103; translation: Math. Notes 46 (1989), no. 5-6, 863-870.

4. C. H. Sah, Cohomology of split group extensions, J. Algebra 29 (1974), 255-302.

5. C. H. Sah, Cohomology of split group extensions II, J. Algebra 45 (1977), 17-68. 